\title{
Identification of Virus in Microscopic Image Using Genetic Algorithm
}

\author{
N. Senthilkumaran ${ }^{1}$ and R. Preethi ${ }^{2}$ \\ ${ }^{1 \& 2}$ Department of Computer Science and Application, \\ The Gandhigram Rural Institute, (Deemed to be University), Dindigul, Tamil Nadu, India \\ E-Mail: preethisengamuthu@gmail.com
}

\begin{abstract}
In this paper describes a several techniques of effective edge detection by using image segmentation. The image segmentation provides various techniques to detect the edges on image. The paper mainly focused on edge detection using matlab parameters and solved the many problems. Edge detection techniques have a several type of techniques. We have taken microscopic image, which affects the human body by making diseases through viruses and bacteria's. Now analyze only about the major techniques: a.) Roberts edge detection, b) sobel edge detection, c) prewitt edge detection, d) log (laplacian of gaussian) edge detection, e) genetic edge detection and f) canny edge detection. We have applied above five techniques which are used in edge detection and got a result on microscopic images. Hence, we scope this paper defines and compares the variety of techniques and demand assures the genetic algorithm provides a better performance on edge detection using microscopic image.

Keywords: Edge Detection, Microscopic, Genetic Algorithm, Image Segmentation
\end{abstract}

\section{INTRODUCTION}

Image Segmentation is used to partition a digital image into multiple regions, which represents a set of regions of entire image. The result of this image segmentation is extracted images [1]. All of the pixels in a region are similar with some characteristic or parameter like color, intensity, and texture [2], [3]. Edge detection is a most often used techniques in digital images processing. The outline of the object surface in a scene has some changes in intensity value of an image called edges. This search considers a research to detect a robust edge detection technique. Many edge detection algorithms are available in image processing. This paper gives description, and comparison of result of recent soft computing approaches for edge detection on segmentation.

The paper is organized as follows. Section II provides a general description of the method and the five test data sets that were used. Section III describes the Genetic Algorithm that was performed. Section IV provides the Experiment and Result. Finally, Section V discusses about conclusions.

\section{EDGEDETECTION}

The image segmentation has different kinds of techniques. It is sub divided the image into several parts, the main goal of image segmentation that can be modified and easier to analyze [4]. Image segmentation is specifically used to locate the object and boundaries. The boundaries are called as lines curves etc. Absolutely we can assign each and every pixel defines as label using edge detection techniques.

The result of image segmentation is a set of segments that collectively fulfill and it will cover the total image. A contours line also called isoclines a set of contours describes from the image segmentation [5]. Each of the pixels in a region is corresponding to some characteristics or computed properly. The image processing has the color, intensity, and texture. Adjacent regions are importantly several with similar to the same principle.

Now we discuss about the main concept of edge detection. Edge detection is a highly developed in the image processing. Region boundaries and edge are one of the techniques which are closely related with edge detection using segmentation. We need to find out the edges after it disconnected. The edges consists of several mathematical methods to target at co ordinates in digital image segmentation and also image brightness with changes accuracy are more professionally as discontinuous using various methods of edge detection. These are step detection \& change detection. Edge detection is a tool of image processing, machine vision and computer vision. It mainly focused on feature detection and feature extraction.

\section{A. Roberts Edge Detection}

The Roberts method is used in image processing and computer vision. The Roberts operator is the one of edge detectors technique. Thus operator specifically proposed by Lawrence Roberts [6]. As a various operator, the suggestion is behind with the Roberts operator is to inexact of the magnitude of an image through discrete differentiation which are gained by computing at the sum of squares of various between diagonally on adjacent pixels.

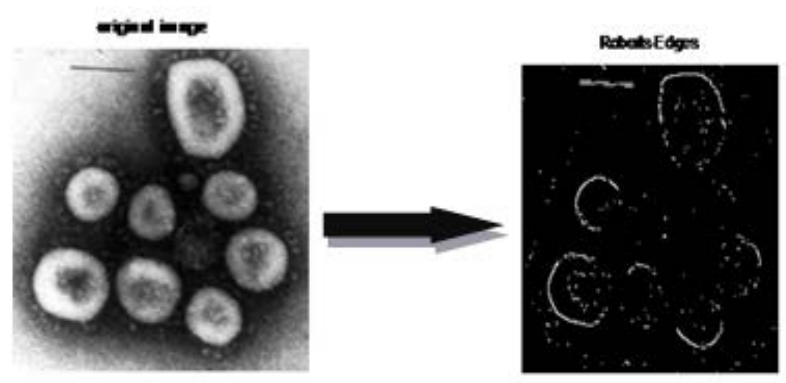

Fig. 1 Corona Virus 


\section{B. Sobel Edge Detection}

In this system are also known as sobel Feldman operator or sobel filter. It is used in the image processing and computer vision [7]. This method is based on convolving the image. The sobel image has small, separable and integer valued filter, occur the horizontal and vertical direction. It is also low expansive in the term of computation, another type the great is approximate to find the relatively crude in a particular high frequency at various image.

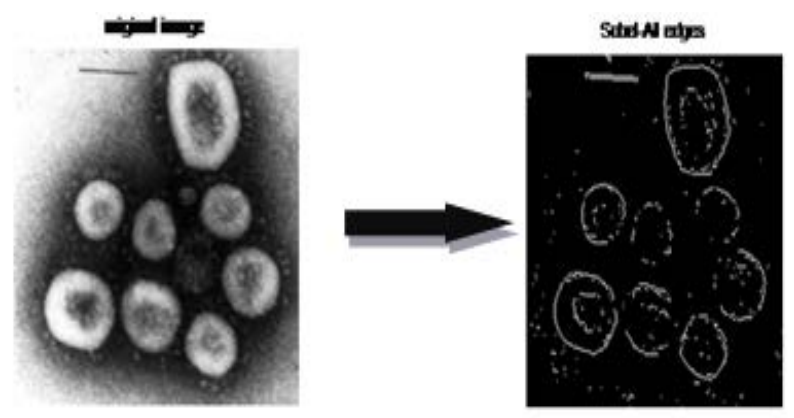

Fig. 2 Corona Virus

\section{Prewitt Edge Detection}

In this operator perform various methods and technique using edge detection. This technique finding image with small portion, separable and integer value filter in horizontal and vertical directions and similarly reasonable in terms of computations like sobel operator, on another type is the grade approximately to find the relatively crude, in the particular for increased frequency difference in an image.
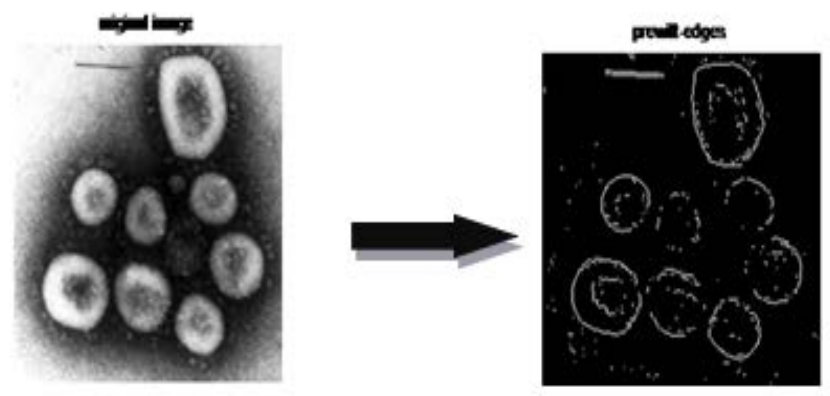

Fig. 3 Corona Virus

\section{LOG (Laplacian of Gaussian) Edge Detection}

An image Laplacian is a 2-Dimensional isotropic measure of the second spatial derivative. The Laplacian of an image indicates the regions of the fast intensity variation and is consequently time and again used for the edge detection. The Laplacian is frequently applied to an image that has the first be curved with something approximate in order to decrease its concern to noise, and therefore the 2 variants will be explained together at here. The operator usually taking a single gray level image as the input and it produces a new gray level image as the output.

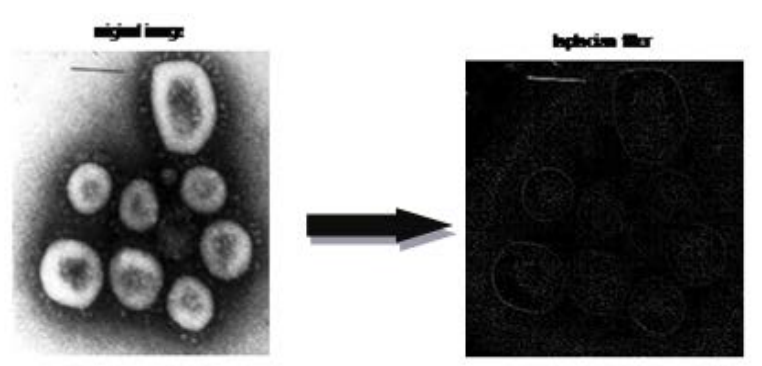

Fig. 4 Corona Virus

\section{E. Canny Edge Detection}

Canny edge detection is a method to extract useful structural in sequence from dissimilar vision objects and considerably decrease the quantity of data to be processed. It has been generally practical in various computer vision systems. Canny has found that the necessities for the application of edge detection on varied vision systems are comparatively related. Hence, an edge detection explanation to concentrate on these requirements can be implemented in a broad variety of the positions.
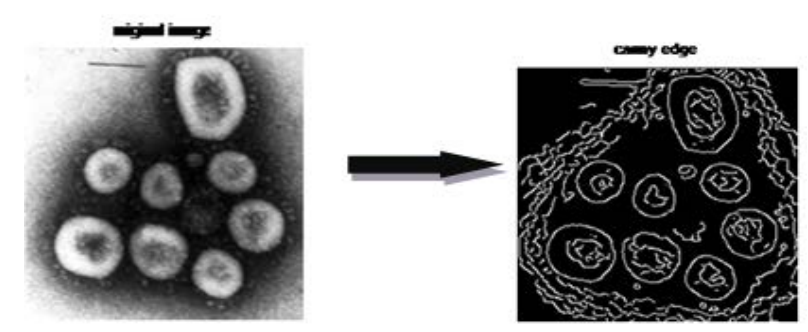

Fig. 5 Corona Virus

\section{GENETIC ALGORITHM FOR EDGE DETECTION}

A Genetic Edge Detection is an operator used in genetic algorithms to show the algorithm towards a solution to a given problem. There are three main types of operatives are mutation, crossover and selection [8]. It has to work in the combination among one an additional in an organizer of the algorithm to be successful. Genetic operators are used to generate and continue genetic different are mutation operator, that merge obtainable solutions is also known as chromosomes and they have the new solutions is crossover and choose between solutions is selections [9], [10]. However, the efficiency of this operator has never been convincingly established and this operator is rarely discussed.

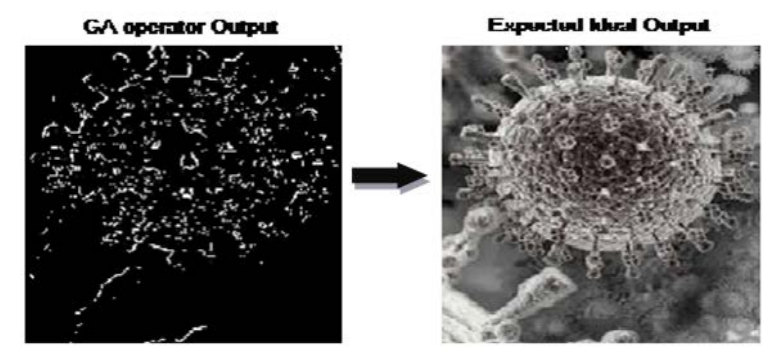

Fig. 6 Bacteria Virus 
Aim: Get a ideal operator which can identify the edges better

Database: Reality edge detection images (input \& ideal output images pair)

Technique: Operator masking based image edge detection Method: Genetic Algorithm

Constraint: Sum of all essentials operator is zero Steps:
1. Load the input image and ideal, expected output image.

2. Apply GA algorithm and find a operator.

3. Present edge detection on input image using above operator.

4. Compare result obtained with ideal expected output using

5. Display the result

\section{EXPERIMENT AND RESULTS}

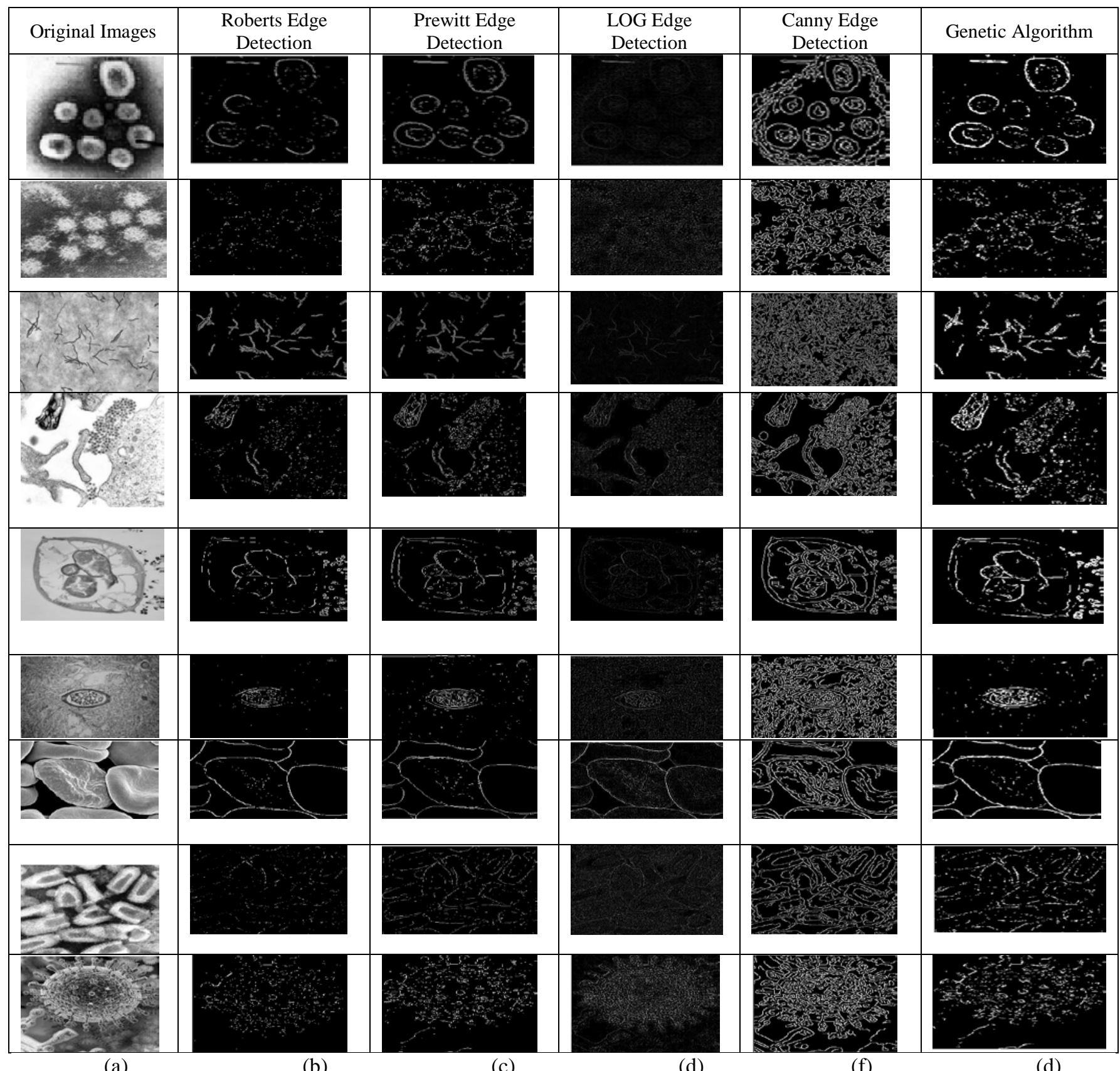

(a)

(b)

(c)

(d)

(f)

(d)

Fig. 7 Edge Detection Techniques (a) Original Image (b) Using Roberts Edge detection (c) Using Prewitt Edge detection (d) Using LOG Edge detection (e) Using Genetic Algorithm 


\section{CONCLUSION}

In this paper, we have used several techniques for edge detection in microscopic images segmentation. We have segmented the microscopic images using Roberts edge detection, Sobel edge detection, Prewitt edge detection, LOG (Laplacian of Gaussian) edge detection, Canny edge detection and Genetic algorithm Edge Detection which are standard edge detection techniques. We have also segmented the images using GA. Finally, we compared the standard edge detection techniques with GA image segmentation. We inferred produces the better result when compared to standard edge detection techniques.

\section{REFERENCES}

[1] P. Moeskops, M. A. Viergever, A. M. Mendrik, L. S. De Vries, M. J. Benders and I. Išgum, "Automatic segmentation of MR brain images with a convolutional neural network", IEEE transactions on medical imaging. Vol. 35, No. 5, pp. 1252-61, May 2016

[2] A. Gooya, K.M. Pohl, M. Bilello, L. Cirillo, G. Biros, E.R. Melhem, and C. Davatzikos, "GLISTR: glioma image segmentation and registration”, IEEE transactions on medical imaging, Vol.31, No. 10, pp. 1941-54, Oct. 2012.

[3] Antonios Makropoulos, Ioannis S. Gousias, Christian Ledig, Paul Aljabar, Ahmed Serag, Joseph V. Hajnal, A. David Edwards, Serena J. Counsell, and Daniel Rueckert, "Automatic Whole Brain MRI
Segmentation of the Developing Neonatal Brain”, IEEE transactions on medical imaging, Vol. 33, No. 9, September 2014.

[4] S. Lakshmi and D.V. Sankaranarayanan, "A study of edge detection techniques for segmentation computing approaches”, IJCA Special Issue on "Computer Aided Soft Computing Techniques for Imaging and Biomedical Applications”, CASCT, Vol. 20, pp. 35-40, Aug. 2010.

[5] N. Senthilkumaran and R. Rajesh, "Edge Detection Techniques for Image Segmentation - A Survey of Soft Computing Approaches", International Journal of Recent Trends in Engineering, Vol. 1, No. 2, May 2009.

[6] Y. Ramadevi, T. Sridevi, B. Poornima, and B. Kalyani, "Segmentation and object recognition using edge detection techniques" International Journal of Computer Science \& Information Technology (IJCSIT), Vol. 2, No. 6, Dec. 2010.

[7] A. Rosenfeld, "The Max Roberts Operator is a Hueckel-Type Edge Detector”, IEEE Transactions on Pattern Analysis and Machine Intelligence, PAMI, Vol. 3, No. 1, pp. 101-103, February 1981.

[8] T. Singh, N. Kharma, M. Daoud, and R.Ward, "Genetic programming based image segmentation with applications to biomedical object detection", In Proceedings of the 11th Annual conference on Genetic and evolutionary computation, ACM, pp. 1123-1130, 8 Jul 2009.

[9] A. Sheta, M.S. Braik, and S. Aljahdali, "Genetic algorithms: a tool for image segmentation”, In 2012 IEEE, International Conference on Multimedia Computing and Systems, pp. 84-90, May 10, 2012.

[10] K.R. Hole, V.S. Gulhane, N.D. Shellokar, "Application of genetic algorithm for image enhancement and segmentation”, International Journal of Advanced Research in Computer Engineering \& Technology (IJARCET), Vol. 2, No. 4, pp. 1342, Apr. 2013. 